\title{
BLIND DECONVOLUTION FOR DIFFRACTION-LIMITED FLUORESCENCE MICROSCOPY
}

\author{
Praveen Pankajakshan ${ }^{1}$, Bo Zhang ${ }^{2}$, Laure Blanc-Féraud ${ }^{1}, Z_{\text {Zi Kam }}^{3}$, \\ Jean-Christophe Olivo-Marin ${ }^{2}$, Josiane Zerubia ${ }^{1}$ \\ ${ }^{1}$ ARIANA Project-team, INRIA/CNRS/UNSA, \\ 2004 route des Lucioles, B.P. 93, \\ 06902 Sophia Antipolis Cedex, France \\ Email: FirstName.LastName@sophia.inria.fr \\ ${ }^{2}$ Quantitative Image Analysis Unit, \\ Institut Pasteur, \\ 25-28 rue du Docteur Roux, \\ 75724 Paris Cedex 15, France \\ ${ }^{3}$ Department of Molecular Cell Biology, \\ Weizmann Institute of Science, \\ Rehovot 76100, Israel
}

\begin{abstract}
Optical Sections of biological samples obtained from a fluorescence Confocal Laser Scanning Microscopes (CLSM) are often degraded by out-of-focus blur and photon counting noise. Such physical constraints on the observation are a result of the diffraction-limited nature of the optical system, and the reduced amount of light detected by the photomultiplier respectively. Hence, the image stacks can benefit from postprocessing restoration methods based on deconvolution. The parameters of the acquisition system's Point Spread Function (PSF) may vary during the course of experimentation, and so they have to be estimated directly from the observation data. We describe here an alternate minimization algorithm for the simultaneous blind estimation of the specimen 3D distribution of fluorescent sources and the PSF. Experimental results on real data show that the algorithm provides very good deconvolution results in comparison to theoretical microscope PSF models.
\end{abstract}

Index Terms - confocal microscopy, blind deconvolution, point spread function, Richardson-Lucy algorithm, total variation regularization

\section{INTRODUCTION}

The CLSM is an optical fluorescence microscope that scans a biological specimen using a focused laser spot and uses a pinhole before the detection to reject the out-of-focus fluorescence [1]. The focusing of the objective lens when done at different depths of the specimen allows 3D visualization of the cells, tissues and embryos removing the need for physical sectioning.

The optics of any observation system allows inspection of

This research was supported by the P2R Franco-Israeli Collaborative Program. The authors gratefully acknowledge Dr. Caroline Chaux (University of Paris-Est) and Prof. Arie Feuer (Technion, Israel) for several interesting discussions. We would also like to thank INRIA for supporting the $\mathrm{PhD}$ of the first author through a CORDI fellowship, CNRS for supporting the $\mathrm{PhD}$ of the second author, and the UMR 6543 from CNRS/UNSA for providing the data. an object, but the image finally obtained is often not perfect. Mathematically the process of degradation is characterized by the PSF, which models the propagation and recording of the electromagnetic radiation from a point source. The PSF thus displays a radial diffractive ring pattern [2] (expanding with defocus) that is introduced by the finite-lens aperture. Thus each optical section has also the out-of-focus contributions from other parts of the object. Although this contribution is greatly reduced in confocal images, it is not totally eliminated, and is dependent on the pinhole diameter.

The main difficulty in restoring the 3D image is that the exact PSF is not precisely known, denoising the image can induce artifacts and restoration by deconvolution is an ill-posed problem. There are three methods to obtain the microscope PSF for the deconvolution process. Firstly, point-like objects in the specimen may be imaged and processed to obtain the PSF. This experimental PSF [3] is in itself dim (therefore may be recorded only at a finite range of defocus values), is contaminated by noise and requires point-like sources in every image. In addition, PSF measured in one sample (typically fluorescent microspheres stuck to a cover slide) may not represent the exact PSF applicable for another sample (such as live cells in physiological buffers). For the second case, when an analytical model [4] is used, the PSF generated is noise free. However, the optical parameters of the set up (for example small residual phase aberrations in the objective) are not known or might change during the course of an experimentation (for example, due to heating of live samples). Thus, we propose a blind deconvolution method based on the third approach that reconstructs the closest approximation of the specimen structure and estimation of the unknown PSF parameters from the observation simultaneously.

\section{JOINT BLUR AND OBJECT ESTIMATION}

\subsection{Image Formation Model}

The detector of the fluorescence microscope behaves ideally as a photon counter (ignoring electronic amplification noise). 
For low illumination conditions, the number of photons reaching the detector is small, and the statistical variation in the number of detected photons can be described by a Poisson process. If $o$ and $i$ denote the original and observed images respectively, the image degradation model can be expressed as,

$$
i(\mathbf{x})=P([h * o](\mathbf{x}))
$$

The object intensity is defined as $\{o(\mathbf{x}): \mathbf{x} \in \Omega\}$, where $\Omega$ is the region over which the intensity is non-vanishing and is finite in nature. Here, $h(\cdot)$ is the PSF of the microscope, and $(*)$ denotes the convolution operator (assuming linearity property of the imaging system).

\subsection{Joint Maximum-Likelihood Estimation}

Since the general noise distortion is assumed to be dominated by Poisson noise, the observation of a fluorescence object $(X=o)$ can be modeled as [5]:

$$
P(Y=i \mid X=o, H=h)=\prod_{\mathbf{x} \in \Omega} \frac{[h * o](\mathbf{x})^{i(\mathbf{x})} e^{-[h * o](\mathbf{x})}}{i(\mathbf{x}) !}
$$

The maximum of this joint likelihood can be found as:

$$
\begin{aligned}
(\hat{o}, \hat{h}) & =\underset{(o, h)}{\arg \max } P(Y=i \mid X=o, H=h) \\
& =\underset{(o, h)}{\arg \min }(-\log [P(Y=i \mid X=o, H=h)])
\end{aligned}
$$

It is easy to see that direct minimization of (3) can yield many possible solutions for $o$ and $h$ (for example, the observed data and an impulse function is one such solution), and their simultaneous estimate from (3) is difficult. Hence, we introduce some beliefs on the object and the PSF to confine the solution space.

\subsection{A priori object model and Total Variation (TV) reg- ularization (Rudin et al. [6])}

The Gibbsian distribution $P(X=o)$, captures the prior knowledge of the object, and is the regularization model. $(X=o)$ is a low-order, homogeneous, isotropic Markov Random Field (MRF), over a 6 member neighborhood $\eta_{\mathbf{x}} \in$ $V_{\mathbf{x}}$ of the site $\mathrm{x} \in \Omega$ (for $3 \mathrm{D}$ images)

$$
\begin{array}{r}
P(X=o) \propto \frac{1}{Z_{\lambda}} e^{-\lambda \sum_{\mathbf{x} \in \Omega}|\nabla o(\mathbf{x})|} \\
\text { where }|\nabla o(\mathbf{x})|=\left(\sum_{\mathbf{x}^{\prime} \in \eta_{\mathbf{x}}}\left(o(\mathbf{x})-o\left(\mathbf{x}^{\prime}\right)\right)^{2}\right)^{\frac{1}{2}} \\
\text { and } Z_{\lambda}=\sum_{o \in \Lambda} e^{-\lambda \sum_{\mathbf{x} \in \Omega}|\nabla o(\mathbf{x})|}
\end{array}
$$

$\Lambda$ is a finite set of possible specimen solutions, $\lambda$ is the global hyperparameter, $\eta_{\mathbf{x}}$ is the set of all cliques for the neighborhood system $V_{\mathbf{x}}$ over the lattice $L .|\nabla o(\mathbf{x})|$ is the potential function associated with clique and $Z_{\lambda}$ is a normalizing constant called the partition function.

\subsection{D Gaussian PSF model}

By using a model for the microscope image acquisition physical process, we can greatly reduce the number of free parameters describing the PSF and thus narrow down the solution space. It was shown in [7] that the CLSM PSF is well modeled by a 3D Gaussian function as:

$$
h_{\sigma_{\rho}, \sigma_{z}}(\rho, z)=\frac{1}{Z_{\sigma_{\rho}, \sigma_{z}}} e^{\left(\frac{-\rho^{2}}{2 \sigma_{\rho}^{2}}-\frac{z^{2}}{2 \sigma_{z}^{2}}\right)}
$$

where $\rho=\sqrt{\left(x^{2}+y^{2}\right)}$ and $Z_{\sigma_{\rho}, \sigma_{z}}=(2 \pi)^{\frac{3}{2}}|\Sigma|^{\frac{1}{2}}$.

A diffraction-limited PSF has a circular symmetry about the $z$-axis and mirror symmetry about the central $x y$-plane. Thus, the covariance matrix should be diagonal [7] and $|\Sigma|=\sigma_{\rho}^{4} \sigma_{z}^{2}$.

\subsection{Joint Maximum A Posteriori estimation}

Therefore the posterior probability $P(X=o, H=h \mid Y=i)$ is given as:

$$
\begin{array}{r}
P(X=o, H=h \mid Y=i) \propto \frac{e^{-\lambda \sum_{\mathbf{x}}|\nabla o(\mathbf{x})|}}{Z_{\lambda}} . \\
\prod_{\mathbf{x} \in \Omega} \frac{[h(\boldsymbol{\theta}) * o](\mathbf{x})^{i(\mathbf{x})} e^{-[h(\boldsymbol{\theta}) * o](\mathbf{x})}}{i(\mathbf{x}) !}
\end{array}
$$

where, $\boldsymbol{\theta}=\left(\sigma_{\rho}, \sigma_{z}\right)$. Thus, the cost function to be minimized is:

$$
\begin{array}{r}
\mathcal{L}(o, h(\boldsymbol{\theta}))=\lambda \sum_{\mathbf{x} \in \Omega}|\nabla o(\mathbf{x})|+\log \left[Z_{\lambda}\right] \\
+\sum_{\mathbf{x} \in \Omega}[h(\boldsymbol{\theta}) * o](\mathbf{x})-\sum_{\mathbf{x} \in \Omega} i(\mathbf{x}) \log [h(\boldsymbol{\theta}) * o](\mathbf{x})
\end{array}
$$

\subsubsection{Object restoration}

Given the initial value (or estimate) of $\hat{\boldsymbol{\theta}},(7)$ can be solved for the object $o$ by adopting the TV regularization, and the Richardson-Lucy (RL) ([4]) algorithm, which is an expectation-maximization algorithm [8] for computing the ML estimate. When the relative difference measure between the successive iterations is smaller than a threshold $(t)$ (see [2]), we stop the algorithm to get the final deconvolved image.

\subsubsection{Parameter estimation on complete data}

Due to the invariance property of ML estimation, we can say that,

$$
\hat{h}_{M L}(\mathbf{x})=h\left(\mathbf{x}, \hat{\boldsymbol{\theta}}_{M L}\right)
$$


is the ML estimate of the PSF. The method outlined in Section 2.5.1 requires the knowledge of parameters $\theta$. This can be obtained by minimizing the following function,

$$
\mathcal{L}(\boldsymbol{\theta} \mid i, \hat{o}) \approx-\sum_{\mathbf{x} \in \Omega}(i(\mathbf{x}) \log [h(\boldsymbol{\theta}) * \hat{o}](\mathbf{x}))+\sum_{\mathbf{x} \in \Omega}[h(\boldsymbol{\theta}) * \hat{o}](\mathbf{x})
$$

The terms that are independent of $\boldsymbol{\theta}$ have been excluded. We now use a gradient based algorithm to estimate $\boldsymbol{\theta}$. The search direction involves calculating $\nabla_{\boldsymbol{\theta}} \mathcal{L}(\boldsymbol{\theta})$ and it is given as:

$$
\begin{array}{r}
\nabla_{\boldsymbol{\theta}} \mathcal{L}(\boldsymbol{\theta})=\sum_{\mathbf{x} \in \Omega}\left(\left(h_{\boldsymbol{\theta}} * \hat{o}\right)(\mathbf{x})-\frac{i(\mathbf{x})}{(h(\boldsymbol{\theta}) * \hat{o})(\mathbf{x})} .\right. \\
\left.\left(h_{\boldsymbol{\theta}} * \hat{o}\right)(\mathbf{x})\right)
\end{array}
$$

where, $h_{\boldsymbol{\theta}}=\frac{\partial}{\partial \boldsymbol{\theta}} h(\boldsymbol{\theta})$. We stop the computation if the relative difference measure between the successive iterations is smaller than a specific threshold $\epsilon$ (in practice $10^{-4}$ ), and assume the last estimate as the best solution.

The starting values for both the object and the parameters are critical as the cost function 7 is not convex w.r.t them. For the object we can simply assume here that the observation is the closest approximation we find to the object. However, for the parameters, this is not straightforward. Since the only condition that has to be satisfied is that the initial states lie outside the solution space, we start with large values and decrease.

\section{RESULTS}

In this section, we present the application of the proposed alternate minimization method to restore real data.

\subsection{Imaging Setup}

The Zeiss LSM 510 confocal microscope mounted on a motorized inverted stand (Zeiss Axiovert 200M) equipped with an $\mathrm{ArKr}$ laser of wavelength of $488 \mathrm{~nm}$ is the excitation source, and Band Pass (BP) filter transmits emitted light within the band $505-550 \mathrm{~nm}$. The objective lens arrangement is a Plan-Neofluar with $40 \mathrm{X}$ magnification having a Numerical Aperture of 1.3 and immersed in oil. The pinhole size was fixed at $67 \mu \mathrm{m}$

\subsection{Biological Specimen}

The biological specimen that was chosen for the experiments is the embryo of a Drosophila melanogaster (see Fig. 1). It was mounted and stained with the Green Fluorescence Protein (GFP), and is useful for studying the sealing of the epithelial sheets (Dorsal Closure) midway during the embryogenesis. The images were acquired with a XY pixel size of $50 \mathrm{~nm}$ and a $\mathrm{Z}$ step size of $170 \mathrm{~nm}$, and the size of the volume imaged is $25.59 \times 25.59 \times 2.55 \mu \mathrm{m}$.

The maximum intensity projection of the observed and restored data is shown in Fig. 1. Since the observation data had
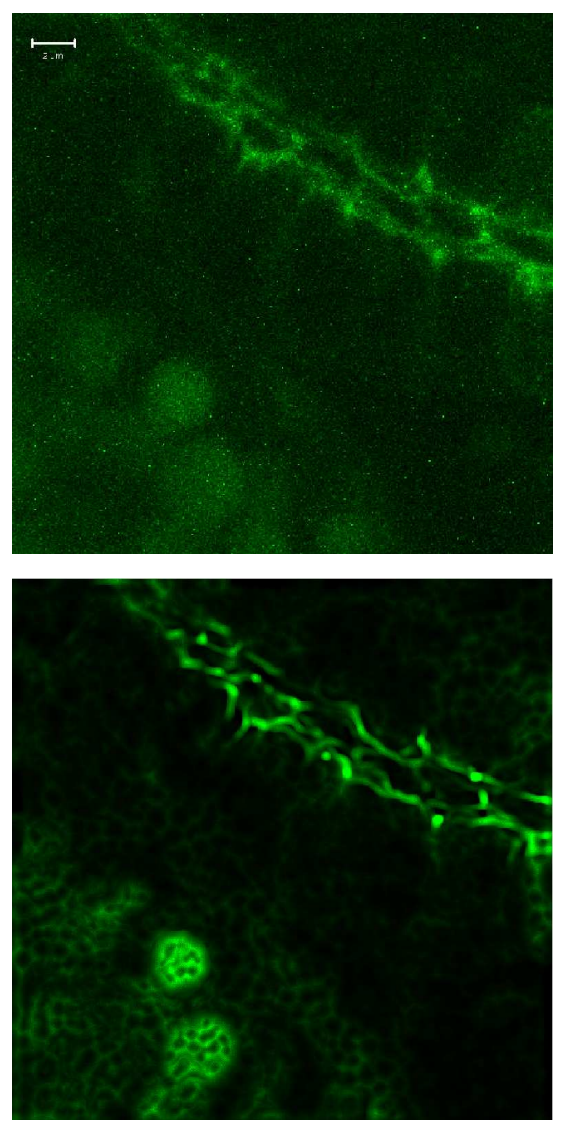

Fig. 1. Maximum Intensity Projection of the original specimen (top) (c) Institute of Signaling, Developmental Biology \& Cancer UMR6543/CNRS/UNSA), and restored image (bottom) (c) INRIA). The intensity is scaled to [0 130] for display and white bar is $2 \mu m$.

a high noise content, the regularization hyperparamter $\lambda$ was initialized to a large value of 0.05 . The deconvolution algorithm was stopped when the difference between subsequent estimates was lower than $t=0.002$. The alternate minimization algorithm converged after 40 iterations of the RLTV algorithm. The PSF parameters were initialized to $300 \mathrm{~nm}$ and $600 \mathrm{~nm}$ for the radial and the axial case respectively, and the conjugate-gradient algorithm estimated them to be really 257.9 and $477.9 \mathrm{~nm}$. These are much larger than their corresponding theoretically expected values [7] [2]. It was verified that the proposed algorithm can not only estimate the actual PSF from the experiments on synthetic data [2], but also provide much better deconvolution results in comparison to theoretical microscope PSF's (generated using the microscope settings).

It was noticed that in each slice of the chosen observation data, there was a significant amount of signal contributions from the neighboring slices too. A subjective analysis of the deconvolution results showed that although (see Fig. 2) some 
structures and cellular walls are visible at $0 \mu \mathrm{m}$ depth of the original data, they are really absent at this level and appears only at $0.34 \mu \mathrm{m}$ in the restoration. The algorithm provides reasonably good deconvolution results even at depths of more than $1 \mu \mathrm{m}$ as shown in Fig. 3.
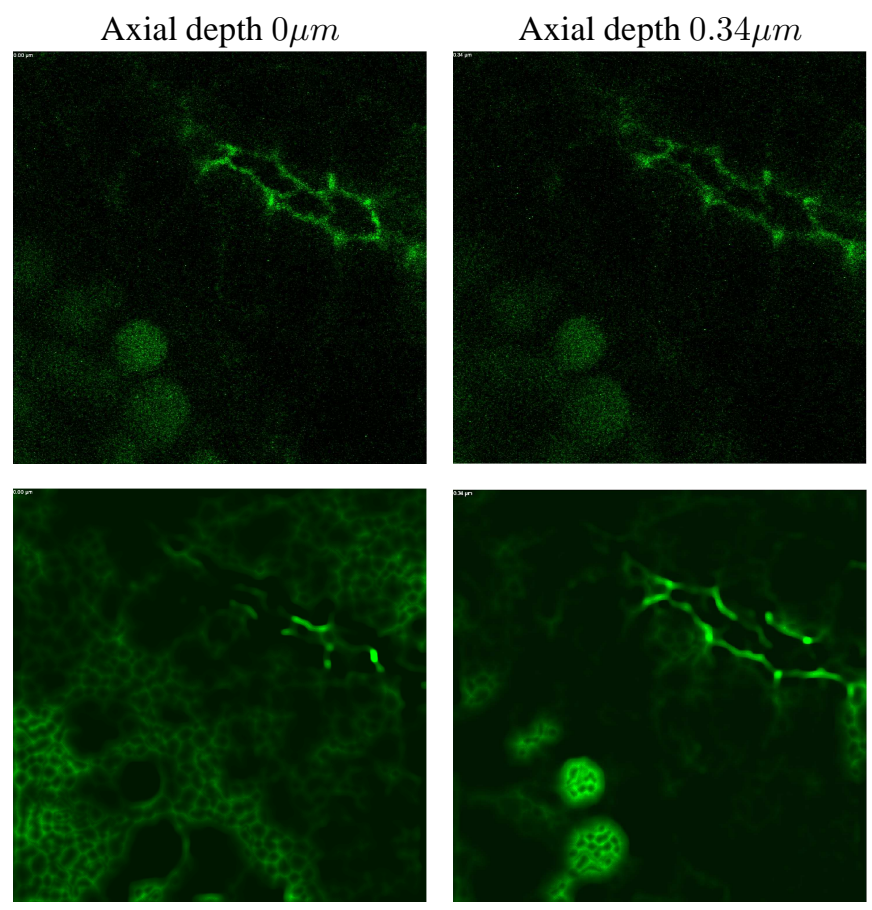

Fig. 2. The observed image slices (top) (c) Institute of Signaling, Developmental Biology \& Cancer UMR6543/CNRS/UNSA) and restoration results (bottom) (C) INRIA) are shown at different depths. The intensity is scaled to [0 100] for display

\section{CONCLUSIONS AND FUTURE WORK}

In this paper we have applied the alternate minimization algorithm to the joint estimation of the microscope PSF and the specimen source distribution. We choose the RL algorithm for the deconvolution process as it is best suited for the Poisson data, and TV as the regularization model. A separable 3D Gaussian model best describes the PSF, and is chosen as the a priori model. We have shown some experimental results on real data, and the method gives very good deconvolution and a PSF estimation closer to the true value [2]. A way to minimize the deblurring artifacts may be to estimate the hyperparameter of the regularization model. Future work is aimed at extending this algorithm to restore spherically aberrated observation data and also to improve the representation of the object to match the physical conditions.

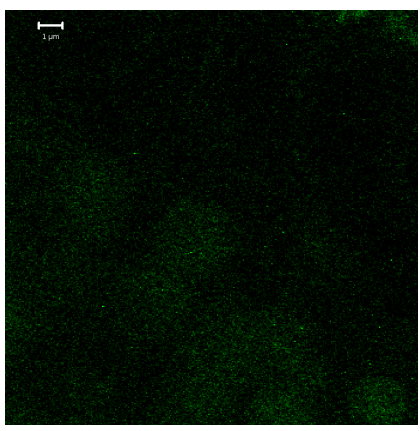

(a)

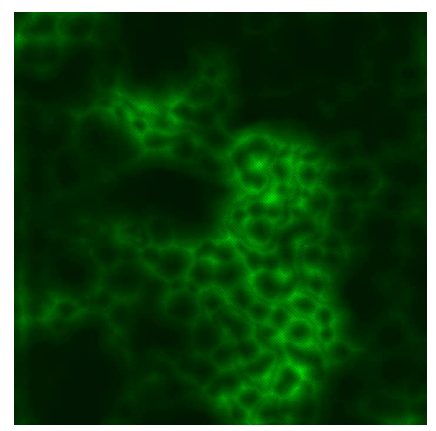

(b)
Fig. 3. Deconvolution results at a depth of $1.53 \mu \mathrm{m}$ (white bar is $1 \mu \mathrm{m}$ ). (a) Observed image section (c) Institute of Signaling, Developmental Biology \& Cancer UMR6543/CNRS/UNSA), (b) Restored image (c) INRIA)

\section{REFERENCES}

[1] J. B. Pawley, Handbook of biological confocal microscopy, Plenum Press, New York, $2^{\text {nd }}$ edition, 1996.

[2] P. Pankajakshan, B. Zhang, L. Blanc-Féraud, Z. Kam, J.C. Olivo-Marin, and J. Zerubia, "Parametric blind deconvolution for confocal laser scanning microscopy," in Proc. 29th International Conference of IEEE EMBS (EMBC-07), Lyon, France, August 2007.

[3] J. G. McNally, C. Preza, J.-Á. Conchello, and L. J. Thomas, "Artifacts in computational optical-sectioning microscopy," Journal of the Optical Society of America A, vol. 11, pp. 1056-1067, Mar. 1994.

[4] N. Dey, L. Blanc-Féraud, C. Zimmer, Z. Kam, P. Roux, J.C. Olivo-Marin, and J. Zerubia, "Richardson-Lucy Algorithm with Total Variation Regularization for 3D Confocal Microscope Deconvolution," Microscopy Research Technique, vol. 69, pp. 260-266, 2006.

[5] D. L. Snyder and M. I. Miller, Random Point Processes in Time and Space, Springer-Verlag, New York, 2nd edition, 1991.

[6] L. I. Rudin, S. Osher, and E. Fatemi, "Nonlinear total variation based noise removal algorithms," Physica D., vol. 60, pp. 259-268, 1992.

[7] B. Zhang, J. Zerubia, and J. C. Olivo-Marin, "Gaussian approximations of fluorescence microscope point spread function models," Applied Optics, vol. 46, no. 10, pp. 1819-1829, 2007.

[8] A. P. Dempster, N. M. Laird, and D. B. Rubin, “Maximum Likelihood from Incomplete Data via the EM Algorithm," Journal of the Royal Statistical Society B, vol. 39, no. 1, pp. 1-38, 1977. 